\title{
Optimization of the Separation of NDA-Derivatized Methylarginines by Capillary and Microchip Electrophoresis
}

\author{
Thomas H. Linz ${ }^{1,2}$, Christa M. Snyder ${ }^{1,3}$, and Susan M. Lunte ${ }^{1,2}$ \\ ${ }^{1}$ Department of Chemistry, University of Kansas, Lawrence, Kansas, USA \\ ${ }^{2}$ Ralph N. Adams Institute for Bioanalytical Chemistry, University of Kansas, Lawrence, Kansas, \\ USA \\ ${ }^{3}$ Wittenberg University, Springfield, Ohio, USA
}

\begin{abstract}
The methylated arginines (MAs) monomethylarginine (MMA), asymmetric dimethylarginine (ADMA), and symmetric dimethylarginine (SDMA) have been shown to be independent predictors of cardiovascular disease. This article describes progress regarding the development of an analytical method capable of rapidly analyzing MAs using capillary electrophoresis (CE) and microchip electrophoresis (MCE) with laser-induced fluorescence (LIF) detection. Several parameters including buffer composition and separation voltage were optimized to achieve an ideal separation. The analytes of interest were derivatized with naphthalene-2,3-dicarboxaldehyde (NDA) to produce fluorescent 1-cyanobenz[f]isoindole (CBI) derivatives and then subjected to $\mathrm{CE}$ analysis. Baseline resolution of SDMA, ADMA, MMA, and arginine was achieved in less than 8 min. The limits of detection for SDMA, ADMA, MMA, and arginine were determined to be 15, 20,25 , and $5 \mathrm{nM}$, respectively, which are well below the expected plasma concentrations. The CE separation method was then transferred to a glass MCE device with LIF detection. MAs were baseline resolved in $3 \mathrm{~min}$ on-chip using a $14 \mathrm{~cm}$ separation channel with detection limits of approximately $10 \mathrm{nM}$ for each species. To the best of the authors' knowledge, this is the first report of the separation of MAs by MCE.
\end{abstract}

\section{Keywords}

methylarginine; capillary electrophoresis; microchip electrophoresis; cardiovascular disease; naphthalenedialdehyde

Cardiovascular disease affects 80 million people in the United States and is the leading cause of death in the nation. ${ }^{1}$ Although there are many factors that contribute to the onset of cardiovascular disease, disruption of the endothelium is believed to play a central role. Nitric oxide (NO) is a crucial molecule for maintaining proper endothelial function., ${ }^{2,3}$ Because it is a small gaseous molecule, NO is capable of rapidly diffusing between cells and can cause physiological responses distal to the site of production. These responses include vasodilation, cellular signaling, and neurotransmission. ${ }^{2}$ It is thought that the reduced

\footnotetext{
(C) 2012 Society for Laboratory Automation and Screening

Corresponding Author: Susan M. Lunte, University of Kansas, Adams Institute, 2030 Becker Drive, Lawrence, KS 66047, slunte@ku.edu.

Christa M. Snyder is a 2011 SLAS Young Scientist Award winner.

Declaration of Conflicting Interests

The authors declared no potential conflicts of interest with respect to the research, authorship, and/or publication of this article.
} 
bioavailability of NO and its subsequent impact on the endothelium is responsible for the development of cardiovascular diseases. ${ }^{2-4}$

The synthesis of NO is carried out by a family of enzymes called nitric oxide synthases (NOSs). These enzymes catalyze the oxidation of arginine to produce citrulline and NO. ${ }^{5}$ Certain methylated arginines are competitive inhibitors of NOS and prevent NO from being produced. ${ }^{6}$ Recent literature has shown that increased levels of these methylarginines (MAs) have been found in patients suffering from pathologies such as stroke, ${ }^{7}$ various heart diseases, ${ }^{8-15}$ and renal failure. ${ }^{16,17}$ The biochemical mechanisms behind the progression of these diseases are not yet well understood, but all have been linked to disrupted endothelium function. Given that MAs reduce the bioavailability of NO, it has been suggested that an abundance of MAs may be responsible for disease onset and progression.

The compounds asymmetric $\mathrm{N}^{\mathrm{G}}, \mathrm{N}^{\mathrm{G}_{-}}$-dimethylarginine (ADMA) and $\mathrm{N}^{\mathrm{G}}$ monomethylarginine (MMA) mimic the NOS substrate arginine and competitively inhibit NO from being synthesized, whereas symmetric $\mathrm{N}^{\mathrm{G}}, \mathrm{N}^{\prime} \mathrm{G}_{\text {-dimethylarginine (SDMA) does }}$ not. ${ }^{18}$ All MAs, however, compete with arginine for cellular uptake by cationic amino acid transporters and, therefore, all affect the cellular production of NO. ${ }^{19}$ Structures and average plasma concentrations of arginine, ${ }^{10,11,15,20-22}$ ADMA, $7,8,10-13,15,20-22$ MMA, ${ }^{8,20}$ and $\mathrm{SDMA}^{7,8,10,11,15,21,22}$ are shown in Figure 1. Despite the fact that all methylated arginines affect the synthesis of NO, most research in the literature has focused strictly on ADMA. This is predominantly because ADMA is present at higher endogenous concentrations than MMA and because SDMA does not competitively inhibit NOS. A recent study performed at the Cleveland Clinic, however, demonstrated that the most robust independent predictor of coronary artery disease was not the quantity of ADMA present but, rather, the arginine methylation index (ArgMI), which is composed of the sum of the dimethylated species concentrations divided by the concentration of the monomethylated form (Equation 1). ${ }^{8}$ This index accurately predicted the extent of disease progression even without adjusting for traditional risk factors. Because of the potential diagnostic proficiency of MAs, an analytical method capable of analyzing them is necessary. This method must be rapid and relatively inexpensive so that it could be used in future large-scale clinical trials.

Capillary electrophoresis (CE) is a useful analytical technique for the analysis of ionic compounds such as MAs. CE provides relatively fast analyses compared with liquid chromatography and is amenable to miniaturization. Microchip electrophoresis (MCE) operates under the same separation principles as conventional $\mathrm{CE}$ but in a much smaller platform. Microchips are only a fraction of the size, which allows them to use smaller sample and reagent volumes and allows analyses to be performed even more rapidly. The short analysis times afforded by an MCE device provide higher sample throughput than other analytical techniques, which will be necessary for future clinical studies addressing MAs. The present study employed laser-induced fluorescence (LIF) detection because of its unparalleled sensitivity for CE and MCE analyses. ${ }^{23}$

There have been multiple reports in the literature describing the use of $\mathrm{CE}$ in the analysis of MAs, but all have shortcomings. The analysis times of these other CE methods range from 13 to 18 min to resolve each of the MA species. ${ }^{24-27}$ Although this is not extremely long, methods with shorter analysis times would be beneficial when analyzing a large number of clinical samples. Also, despite their relatively long run times, several of the reported methods still suffer from the comigration of the MA compounds. Additional limitations of these other methods stem from their choices of detection techniques. The use of UV detection $^{25}$ is not ideal because of its lack of selectivity at $190 \mathrm{~nm}$ as well as its relatively high detection limits (compared with LIF). The studies that used fluorescence detection in the analysis of MAs chose to use derivatization reagents that hindered sample throughput 
either by necessitating that the labeling reaction take place overnight ${ }^{27}$ or by requiring the sample to be heated for derivatization to occur. ${ }^{26}$ Neither of these approaches is ideal because of the lengthy sample preparation times. In addition, the derivatization reagents used in previous reports are fluorescent themselves. This complicates the separation by requiring the excess derivatization reagent in the reaction mixture to be resolved from the labeled analytes of interest. The CE method in the literature that is most promising uses a tandem mass spectrometer as a detector. Despite having the best reported limits of detection for MAs, ${ }^{24}$ the cost of analysis is quite large due to the high price of a CE-MS/MS system and the need for expensive deuterated internal standards.

The purpose of this study was to develop a fast, relatively inexpensive analytical method for the determination of methylarginines. MA standards were labeled with naphthalene-2,3dicarboxaldehyde (NDA), which is a fluorogenic derivatization reagent. Run conditions were then optimized using $\mathrm{CE}$ with fluorescence detection to determine the conditions necessary to achieve baseline resolution between the four analytes of interest. This separation method was subsequently transferred to an MCE device, where the rapid analysis of MAs was performed. To our best knowledge, this report is the first to demonstrate the separation of MAs on-chip, as well as the CE-LIF method that provides the best limits of detection to date.

\section{Materials and Methods}

\section{Reagents}

Standard solutions of arginine (Sigma Aldrich, St. Louis, MO), ADMA, SDMA, and MMA (Enzo Life Sciences, Plymouth Meeting, PA) were prepared at $10 \mathrm{mM}$ concentrations in 18 $\mathrm{M} \Omega$ deionized water (Millipore, Billerica, MA). Samples were serially diluted to the desired concentrations prior to $\mathrm{CE}$ analysis. A stock solution of $100 \mathrm{mM}$ sodium tetraborate (Sigma Aldrich) was made. Aliquots of the borate solution were adjusted to the indicated $\mathrm{pH}$ values either by the addition of $1 \mathrm{~N} \mathrm{HCl}$ or the addition of $1 \mathrm{~N}$ and/or $10 \mathrm{~N} \mathrm{NaOH}$. A $50 \mathrm{mM}$ aqueous solution of sulfobutylether- $\beta$-cyclodextrin (SBEC) (Cydex Pharmaceuticals, Lenexa, KS) was also prepared. To formulate the run buffers, stock solutions of borate and SBEC were combined and diluted to the appropriate concentrations. NDA (Invitrogen, Carlsbad, CA) was dissolved in 1:1 acetonitrile:water to a final concentration of $5 \mathrm{mM}$. A 10 $\mathrm{mM}$ sodium cyanide (Sigma Aldrich) solution was prepared in water.

\section{Derivatization}

Because MAs are not natively fluorescent, the analytes were derivatized with NDA prior to analysis. NDA labels primary amines in the presence of cyanide to produce fluorescent 1cyanobenz[f]isoindole (CBI) products. The excitation and emission maxima of CBI derivatives are $440 \mathrm{~nm}$ and $490 \mathrm{~nm}$, respectively. LIF detection was chosen for this study because it provides the best detection limits for the determination of CBI derivatives. ${ }^{28,29}$ Samples were derivatized by combining equal volumes of methylarginine standards, 100 $\mathrm{mM} \mathrm{pH} 9.25$ sodium tetraborate, NDA, and sodium cyanide. The mixture was allowed to react for 20 min under ambient conditions and then immediately injected into the $\mathrm{CE}$. New samples were prepared prior to every run. Three samples were analyzed under each set of conditions.

\section{Capillary Electrophoresis}

A Beckman P/ACE MDQ CE instrument (Brea, CA) equipped with a $65 \mathrm{~cm}(50 \mathrm{~cm}$ to window), $50 \mu \mathrm{m}$ i.d. $/ 360 \mu \mathrm{m}$ o.d. fused silica capillary (Polymicro Technologies, Phoenix, AZ) was used in the development of a method to separate the MA species. Samples were injected at $1.0 \mathrm{psi}$ for $5 \mathrm{~s}$. A Picometrics ZetaLIF external fluorescence detector 
(Ramonville, France) with a $445 \mathrm{~nm}$ CW diode laser (CrystaLaser, Reno, NV) was used to detect the analytes. Both the CE parameters and the LIF detection were controlled by 32Karat software.

\section{Microchip Electrophoresis}

The procedure for the fabrication of the glass MCE device used in this article has been previously reported by our lab. ${ }^{30,31}$ Briefly, photolithography was first performed on a borofloat glass substrate coated with layers of chromium and positive photoresist (Telic, Valencia, CA). A photomask (Infinite Graphics, Minneapolis, MN) containing the channel design of interest was aligned over the glass and put into hard contact using a mask aligner (ABM, Scotts Valley, CA). The glass plate was subsequently exposed to UV radiation from the mask aligner and developed using 300 MIF Developer (AZ, Somerville, NJ). After removing the exposed chromium with chromium etchant (Cyantek, Fremont, CA), the glass was etched using a 20:14:66 solution of hydrofluoric acid (Acros Organic, Pittsburgh, PA), nitric acid (Fisher Scientific, Pittsburgh, PA) and water, respectively, to create recessed channels in the glass substrate. Etching continued until a channel depth of $15 \mu \mathrm{m}$ was reached as verified by a profilometer (Tencor, Milpitas, CA). Holes were then drilled in a cover plate of glass to create reservoirs. The cover plate and channel plate were aligned and subjected to a thermal bonding procedure using a programmable furnace (Fisher Scientific). Following the thermal bond, the pieces of glass were fused together and could then be used as an MCE device. The final channel dimensions were $15 \times 45 \mu \mathrm{m}$ with a $14 \mathrm{~cm}$ separation channel ( $13 \mathrm{~cm}$ to detector). The sample reservoir side arm was $1 \mathrm{~cm}$ in length, whereas the buffer and sample waste side arms were $4 \mathrm{~cm}$ long. The microchip channel design is illustrated in Figure 2.

To operate the microchip device, all channels were first filled with run buffer. A few microliters of derivatized sample were then loaded into the sample reservoir, and run buffer was loaded into the buffer, buffer waste, and sample waste reservoirs (see Fig. 2). An UltraVolt HV Rack high-voltage power supply (Ronkonkoma, NY) was used to apply $7 \mathrm{kV}$ to the sample reservoir and $11 \mathrm{kV}$ to the buffer reservoir. The other two reservoirs were held at ground. Applying the voltage in this fashion created a gated injection scheme that forced fluid to flow continuously from the sample reservoir to the sample waste reservoir and from the buffer reservoir to the buffer waste. Injections were made into the separation channel by floating the high voltage in the buffer reservoir for $0.2 \mathrm{~s}$. The high voltage was then reapplied to reestablish gating and also to allow the components of the sample plug to separate as they were electrokinetically driven down the separation channel. A custom program to control the high-voltage application and data collection was written in house using Labview software (National Instruments, Austin, TX).

To collect the fluorescence signal from the microchip device, a $445 \mathrm{~nm}$ PhoxX diode laser (Market Tech, Scotts Valley, CA) was directed into an epifluorescence microscope (Nikon, Melville, NY). The laser spot emanating from the microscope objective was focused onto the MCE device just upstream from the buffer waste reservoir. As NDA-labeled analytes passed across the laser spot, their fluorescent emission passed to a photomultiplier tube, where the response was detected using the aforementioned Labview program.

\section{Results and Discussion}

\section{Buffer Modification}

Several reagents were evaluated as run buffer modifiers in an attempt to separate the MAs (data not shown). Inadequate resolution was observed with free-zone electrophoresis using either borate or a combination of borate and 3-(cyclohexylamino)-1-propanesulfonic acid (CAPS) as a run buffer. Run buffer containing only borate led to complete comigration of 
the analytes of interest. The addition of CAPS into the borate enhanced the resolution and allowed arginine, MMA, and either ADMA or SDMA to be separated; however, the two dimethylarginines still comigrated. In an effort to improve resolution, $\beta$-cyclodextrin was added in an attempt to resolve the two dimethyl species, but ultimately this did not improve the separation. A micellar electrokinetic chromatography (MEKC) approach using sodium dodecyl sulfate (SDS) was also evaluated, but this technique did not adequately resolve the compounds.

Sulfobutylether- $\beta$-cyclodextrin was then evaluated based on a previous study that used it to help resolve -phthaldehyde-labeled biogenic amines. ${ }^{32}$ It was discovered that SBEC provided baseline resolution of our four analytes of interest. SBEC facilitated the separation of NDA-labeled MAs by two mechanisms. ${ }^{33}$ First, the 1-cyanobenz[f]isoindole ring partitioned into the hydrophobic core of the cyclodextrin. Because SBEC is anionic and has a negative electrophoretic mobility, the net migration of the derivatized species was retarded as the molecule interacted with the cyclodextrin ring. Neutral $\beta$-cyclodextrin did not affect the separation because it lacked the negative mobility of SBEC, so there was no significant retention during analyte partitioning. In addition to the interaction with the CBI ring, the cationic guanidinium groups on the arginine residues interacted with the negatively charged sulfate groups on the surface of the SBEC to form an ion pair. The methyl groups on MMA, ADMA, and SDMA, however, attenuated the electrostatic attraction due to steric hindrance, thereby diminishing the retention of the analyte. The extent of methylation and the subsequent inhibition of electrostatic association with the cyclodextrin correctly predicts the migration order of SDMA, ADMA, MMA, and arginine.

\section{Separation Optimization}

The concentrations of borate and SBEC as well as the run buffer $\mathrm{pH}$ and separation voltage were systematically varied to determine the optimal conditions for the separation of $500 \mathrm{nM}$ MAs. The concentration of SBEC was varied from 0 to $20 \mathrm{mM}$ in $5 \mathrm{mM}$ increments to evaluate its effect on the resolution between the analytes. A plot of average migration time versus SBEC concentration is shown in Figure 3. This graph illustrates the increase in resolution with increasing amounts of the anionic cyclodextrin. The MAs were completely unresolved when no SBEC was included in the run buffer. (Data points from these electropherograms were omitted from Fig. 3 for clarity.) It should be noted that complete baseline resolution was achieved at all of the other SBEC concentrations. A run buffer containing $5 \mathrm{mM}$ SBEC was chosen for subsequent analyses because all analytes of interest were resolved from one another in the shortest run times.

Sodium tetraborate is a common background electrolyte used in CE separations. It was selected as the background electrolyte for this analysis because of its compatibility with the NDA reaction mixture. The borate concentration in the run buffer was varied from 10 to 50 $\mathrm{mM}$ to determine the optimal conditions for separating the analytes of interest (Fig. 4). Higher borate concentrations provided better resolution between adjacent peaks at the cost of increased analysis times. Concentrations as low as $10 \mathrm{mM}$ borate still resulted in baseline resolution of the analytes. However, at low borate concentrations, the MAs had migration times near the neutral marker. Although this was not problematic when analyzing MA standards, for the analysis of more complex sample mixtures, additional peak capacity may be needed to completely resolve the peaks of interest from other matrix components. As a result, it was determined that $30 \mathrm{mM}$ borate was optimal. At this concentration, complete baseline resolution between the four MAs was obtained in reasonable run times of $8 \mathrm{~min}$ while having supplementary peak capacity to prevent comigration of potentially interfering species. 
The $\mathrm{pH}$ of the borate solution was also varied to ascertain the impact of $\mathrm{pH}$ on peak resolution. Run buffers consisting of $30 \mathrm{mM}$ borate and $5 \mathrm{mM} \mathrm{SBEC}$ were prepared at $\mathrm{pH}$ 9.00, 9.25, 9.50, 9.75, and 10.00. The effect of $\mathrm{pH}$ on migration time is shown in Figure 5. It can be seen that the higher $\mathrm{pH}$ buffers produced longer migration times. All the compounds were completely resolved regardless of run buffer $\mathrm{pH}$. For simplicity, a pH of 9.25 was selected for subsequent analyses as that is also the optimum $\mathrm{pH}$ at which to derivatize the analytes of interest.

An investigation was also made into the effect of the applied separation voltages on resolution and analysis time. As expected, higher voltages led to faster analyte migration and shorter analysis times. This trend can be seen in Figure 6. As one of the goals of developing this method was to increase throughput, the fastest possible separation is desired. Because all species of interest remained baseline resolved at the higher field strengths, a separation voltage of $28 \mathrm{kV}(430 \mathrm{~V} / \mathrm{cm})$ was determined to be optimal.

The optimized separation parameters were determined after performing a systematic evaluation of the effects of the analysis conditions, as described above. The final run buffer was composed of $30 \mathrm{mM} \mathrm{pH} 9.25$ borate and $5 \mathrm{mM}$ SBEC. A separation voltage of $28 \mathrm{kV}$ was also determined to be ideal. Baseline resolution of all the analytes of interest was achieved with run times of less than 8 min. A sample electropherogram illustrating the optimized separation is shown in Figure 7. The limits of detection (LODs) for each of the MA species were determined using this CE-LIF method. The experimentally determined LODs $(\mathrm{S} / \mathrm{N}=3)$ for arginine, ADMA, MMA, and SDMA were 5, 20, 25, and $15 \mathrm{nM}$, respectively, corresponding to mass detection limits of 10, 40, 50, and 30 amol. It should be noted that all of the detection limits are below the concentrations expected in plasma. This will allow endogenous levels of MAs to be detected and quantified using our method.

\section{Microchip System}

Once the conventional CE method had been optimized, similar separation parameters were evaluated on an MCE device. Because the glass channels of the microchip are analogous in composition to the walls of the silica capillary, the EOF for the two methods should be similar, and a comparable separation should be achieved. The MCE device was operated using the same $30 \mathrm{mM}$ borate/5 $\mathrm{mM}$ SBEC run buffer. To perform a gated injection on a microchip, however, two high-voltage electrodes were required to produce the two different flow streams as opposed to the linear electric field necessary for a conventional CE separation. It was determined that applied voltages of $7 \mathrm{kV}$ and $11 \mathrm{kV}$ were optimal for the sample and buffer reservoirs on the microchip, respectively. Once this optimization was completed, a sample containing $1 \mu \mathrm{M}$ of each NDA-derivatized MA species was loaded into the sample reservoir and analyzed on-chip. An electropherogram depicting that separation is shown in Figure 8. Near-complete baseline resolution was achieved for the MAs on an MCE device in approximately $3 \mathrm{~min}$. The on-chip detection limits $(\mathrm{S} / \mathrm{N}=3)$ for all four analytes of interest were determined to be approximately $10 \mathrm{nM}$. We believe that this is the first report describing the separation of methylarginines on an MCE device. The use of MCE will greatly facilitate the throughput of the analytical method because of the shorter analysis times without sacrificing sensitivity, as evidenced by the low LODs.

Improvements could be made to decrease analysis times by increasing the field strengths in the microchip. In this study, the separation current in the channels was limited to a $100 \mu \mathrm{A}$ maximum. This limit was set as an arbitrary threshold to prevent Joule heating from producing bubbles in the chip. As a result, gating voltages of $7 \mathrm{kV}$ and $11 \mathrm{kV}$ were chosen because they provided the highest field strengths while preventing the current from exceeding the maximum threshold. The $7 / 11 \mathrm{kV}$ voltage scheme corresponded to field strengths of $560 \mathrm{~V} / \mathrm{cm}$. However, field strengths well over $1000 \mathrm{~V} / \mathrm{cm}$ are theoretically 
possible before boiling the buffer. ${ }^{34}$ Because no significant bubble formation occurred under our conditions and because higher electric fields have been reported, higher voltages could

be applied to our chip to help increase the rate of analysis and improve separation efficiency.

\section{Conclusions}

A CE-based separation method was developed for the determination of four methylarginine species involved in cardiovascular disease. A run buffer containing $30 \mathrm{mM} \mathrm{pH} 9.25$ borate and $5 \mathrm{mM} \mathrm{SBEC}$ and a separation voltage of $28 \mathrm{kV}(430 \mathrm{~V} / \mathrm{cm})$ were determined to be optimal run parameters. Baseline resolution was achieved between NDA-derivatized SDMA, ADMA, MMA, and arginine in less than $8 \mathrm{~min}$. The separation method was then transferred to an MCE device where the analytes of interest were able to be separated in 3 min over a $13 \mathrm{~cm}$ separation distance. This is the first report demonstrating the separation of MAs using MCE.

Future work will be conducted to improve the separation of the MAs on-chip by modifying the applied voltages and the run buffer composition. Once sufficient separations are reproducibly achieved, additional separation channels will be incorporated into the device. This will allow several samples to be analyzed simultaneously while maintaining the fast analysis times. Studies are currently underway to develop a solid phase extraction sample preparation procedure to selectively isolate MAs from plasma. When this method is optimized, clinical samples obtained from patients with and without coronary atherosclerosis will be analyzed by MCE. This high-throughput method will allow a large number of samples to be analyzed so that more data can be generated and used to determine the correlation between MAs and the extent of atherosclerosis. This information will help to improve the understanding of the pathways involved in the onset and progression of CVD.

\section{Acknowledgments}

The authors thank Dr. Val Stella for providing the sulfobutylether- $\beta$-cyclodextrin and Nancy Harmony for her assistance in the preparation of this article.

Funding

The authors gratefully acknowledge funding for this work provided by the Adams Institute for Bioanalytical Chemistry, NIH NS61202, and the NSF REU program. They also acknowledge the ASDL and SLAS for providing funding to present this work at LabAutomation 2011.

\section{References}

1. American Heart Association. Cardiovascular Disease Statistics. http://www.americanheart.org/ presenter.jhtml?identifier $=4478$

2. Culotta E, Koshland DE Jr. NO News Is Good News. Science. 1992; 25:1862-1865. [PubMed: 1361684]

3. Dusting GJ. Nitric Oxide in Cardiovascular Disorders. J Vasc Res. 1995; 3:143-161. [PubMed: 7772676]

4. Wennmalm A. Endothelial Nitric Oxide and Cardiovascular Disease. J Intern Med. 1994; 23:317327. [PubMed: 8151263]

5. Marletta MA. Nitric Oxide Synthase Structure and Mechanism. J Biol Chem. 1993; 26:1223112234. [PubMed: 7685338]

6. Vallance P. Importance of Asymmetrical Dimethylarginine in Cardiovascular Risk. Lancet. 2001; 35:2096-2097. [PubMed: 11784617]

7. Leong T, Zylberstein D, Graham I, Lissner L, Ward D, Fogarty J, Bengtsson C, Bjorkelund C, Thelle D. Asymmetric Dimethylarginine Independently Predicts Fatal and Nonfatal Myocardial 
Infarction and Stroke in Women: 24-Year Follow-up of the Population Study of Women in Gothenburg. Arterioscler Thromb Vasc Biol. 2008; 2:961-967. [PubMed: 18292394]

8. Wang Z, Tang WHW, Cho L, Brennan DM, Hazen SL. Targeted Metabolomic Evaluation of Arginine Methylation and Cardiovascular Risks: Potential Mechanisms beyond Nitric Oxide Synthase Inhibition. Arterioscler Thromb Vasc Biol. 2009; 2:1383-1391. [PubMed: 19542023]

9. Goonasekera CDA, Rees DD, Woolard P, Frend A, Shah V, Dillon MJ. Nitric Oxide Synthase Inhibitors and Hypertension in Children and Adolescents. J Hypertens. 1997; 1:901-909. [PubMed: 9280214]

10. Duckelmann C, Mittermayer F, Haider DG, Altenberger J, Eichinger J, Wolzt M. Asymmetric Dimethylarginine Enhances Cardiovascular Risk Prediction in Patients with Chronic Heart Failure. Arterioscler Thromb Vasc Biol. 2007; 2:2037-2042. [PubMed: 17569878]

11. Duckelmann C, Mittermayer F, Haider DG, Altenberger J, Wolzt M. Plasma Asymmetric Dimethylarginine and Cardiovascular Events in Patients with Acute Decompensated Heart Failure. Transl Res. 2008; 15:24-30. [PubMed: 18593634]

12. Schnabel R, Blankenberg S, Lubos E, Lackner KJ, Rupprecht HJ, Espinola-Klein C, Jachmann N, Post F, Peetz D, Bickel C, Cambien F, Tiret L, Munzel T. Asymmetric Dimethylarginine and the Risk of Cardiovascular Events and Death in Patients with Coronary Artery Disease: Results from the AtheroGene Study. Circ Res. 2005; 9:e53-59. [PubMed: 16100045]

13. Miyazaki H, Matsuoka H, Cooke JP, Usui M, Ueda S, Okuda S, Imaizumi T. Endogenous Nitric Oxide Synthase Inhibitor: A Novel Marker of Atherosclerosis. Circulation. 1999; 9:1141-1146. [PubMed: 10069780]

14. Lee L, Webb RC. Endothelium-Dependent Relaxation and L-Arginine Metabolism in Genetic Hypertension. Hypertension. 1992; 1:435-441. [PubMed: 1568761]

15. Zeller M, Korandji C, Guilland JC, Sicard P, Vergely C, Lorgis L, Beer JC, Duvillard L, Lagrost AC, Moreau D, Gambert P, Cottin Y, Rochette L. Impact of Asymmetric Dimethylarginine on Mortality after Acute Myocardial Infarction. Arterioscler Thromb Vasc Biol. 2008; 2:954-960. [PubMed: 18276906]

16. Zoccali C, Bode-Boger SM, Mallamaci F, Benedetto FA, Tripepi G, Malatino LS, Cataliotti A, Bellanuova I, Fermo I, Frolich JC, Boger RH. Plasma Concentration of Asymmetrical Dimethylarginine and Mortality in Patients with End-Satge Renal Disease: A Prospective Study. Lancet. 2001; 35:2113-2117. [PubMed: 11784625]

17. Fleck C, Janz A, Schweitzer F, Karge E, Schwertfeger M, Stein G. Serum Concentrations of Asymmetric (ADMA) Dimethylarginine and Symmetric (SDMA) Dimethylarginine in Renal Failure Patients. Kidney Int. 2001; 5:S14-S18.

18. Tran CTL, Leiper JM, Vallance P. The DDAH/ADMA/ NOS Pathway. Atheroscler Suppl. 2003:33-40. [PubMed: 14664901]

19. Zakrzewicz D, Eickelberg O. From Arginine Methylation to ADMA: A Novel Mechanism with Therapeutic Potential in Chronic Lung Diseases. BMC Pulm Med. 2009:5. [PubMed: 19178698]

20. Chirinos JA, David R, Bralley JA, Zea-Diaz H, Munoz-Atahualpa E, Corrales-Medina F, CubaBustinza C, Chirinos-Pacheco J, Medina-Lezama J. Endogenous Nitric Oxide Synthase Inhibitors, Arterial Hemodynamics, and Subclinical Vascular Disease: The PREVENCION Study. Hypertension. 2008; 5:1051-1059. [PubMed: 18852383]

21. Bulau P, Zakrzewicz D, Kitowska K, Leiper J, Gunther A, Grimminger F, Eickelberg O. Analysis of Methylarginine Metabolism in the Cardiovascular System Identifies the Lung as a Major Source of ADMA. Am J Physiol Lung Cell Mol Physiol. 2007; 29:L18-L24. [PubMed: 16891395]

22. Ajtay Z, Scalera F, Cziraki A, Horvath I, Papp L, Sulyok E, Szabo C, Martens-Lobenhoffer J, Awiszus F, Bode-Boger SM. Stent Placement in Patients with Coronary Heart Disease Decreases Plasma Levels of the Endogenous Nitric Oxide Synthase Inhibitor ADMA. Int J Mol Med. 2009; 2:651-657. [PubMed: 19360324]

23. Bruin GJM. Recent Developments in Electrochemically Driven Analysis on Microfabricated Devices. Electrophoresis. 2000; 2:3931-3951. [PubMed: 11192117]

24. Desiderio C, Rossetti DV, Messana I, Giardina B, Castagnola M. Analysis of Arginine and Methylated Metabolites in Human Plasma by Field Amplified Sample Injection Capillary 
Electrophoresis Tandem Mass Spectrometry. Electrophoresis. 2010; 3:1894-1902. [PubMed: 20432477]

25. Zinellu A, Sotgia S, Scanu B, Formato M, Deiana L, Carru C. Assessment of Protein-Incorporated Arginine Methylation in Biological Specimens by CZE UV-Detection. Electrophoresis. 2007; 2:4452-4458. [PubMed: 17987631]

26. Trapp G, Sydow K, Dulay MT, Chou T, Cooke JP, Zare RN. Capillary Electrophoretic and Micellar Electrokinetic Separations of Asymmetric Dimethyl-L-Arginine and Structurally Related Amino Acids: Quantitation in Human Plasma. J Sep Sci. 2004; 2:1483-1490. [PubMed: 15638156]

27. Causse E, Siri N, Arnal JF, Bayle C, Malatray P, Valdiguie P, Salvayre R, Couderc F. Determination of Asymmetric Dimethylarginine by Capillary Electrophoresis-Laser Induced Fluorescence. J Chromatogr B. 2000; 74:77-83.

28. Lunte SM, Wong OS. Precolumn Derivatization with Naphthalenedialdehyde/Cyanide for Fluorescence, Chemiluminescence or Electrochemical Detection of Primary Amines and Peptides. Curr Sep. 1990; 1:19-25.

29. Lunte SM, Wong OS. Naphthalenedialdehyde-Cyanide: A Versatile Fluorogenic Reagent for the LC Analysis of Peptides and Other Primary Amines. LC-GC. 1989:908-910. 912, 914, 916.

30. Huynh BH, Fogarty BA, Nandi P, Lunte SM. A Micochip Electrophoresis Device with Online Microdialysis Sampling and On-chip Sample Derivatization by Naphthalene 2,3dicarboxaldehyde/2-mercaptoethanol for Amino Acid and Peptide Analysis. J Pharm Biomed Anal. 2006; 4:529-534. [PubMed: 16829012]

31. Coltro WKT, Lunte SM, Carrilho E. Comparison of the Analytical Performance of Electrophoresis Microchannels Fabricated in PDMS, Glass, and Polyester-Toner. Electrophoresis. 2008; 2:49284937. [PubMed: 19025869]

32. Male KB, Luong JH. Derivatization, Stabilization and Detection of Biogenic Amines by Cyclodextrin-Modified Capillary Electrophoresis-Laser-Induced Fluorescence Detection. J Chromatogr A. 2001; 92:309-317. [PubMed: 11556335]

33. Zia V, Rajewski RA, Stella VJ. Effect of Cyclodextrin Charge on Complexation of Neutral and Charged Substrates: Comparison of (SBE)7M- $\beta-C D$ to HP- $\beta-C D$. Pharm Res. 2001; 1:667-673. [PubMed: 11465424]

34. Petersen NJ, Nikolajsen RPH, Mogensen KB, Kutter JP. Effect of Joule Heating on Efficiency and Performance for Microchip-Based and Capillary-Based Electrophoretic Separation Systems: A Closer Look. Electrophoresis. 2004; 2:253-269. [PubMed: 14743478] 


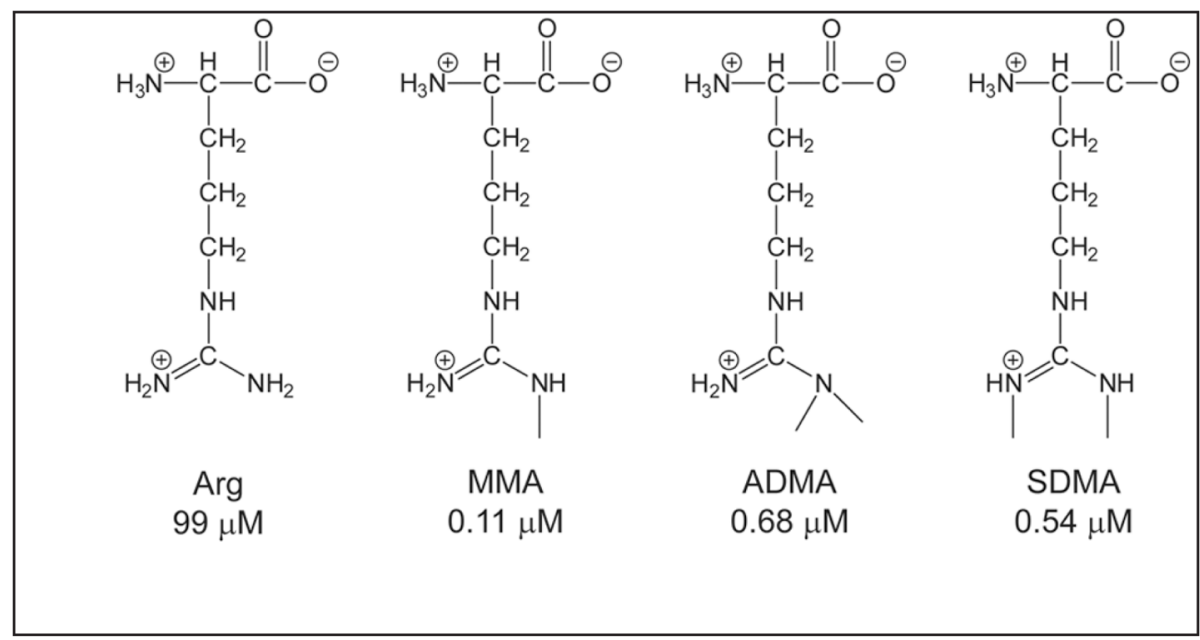

Figure 1.

Structures and average plasma concentrations of the methylarginines. $7,8,10-13,15,20-22$ 


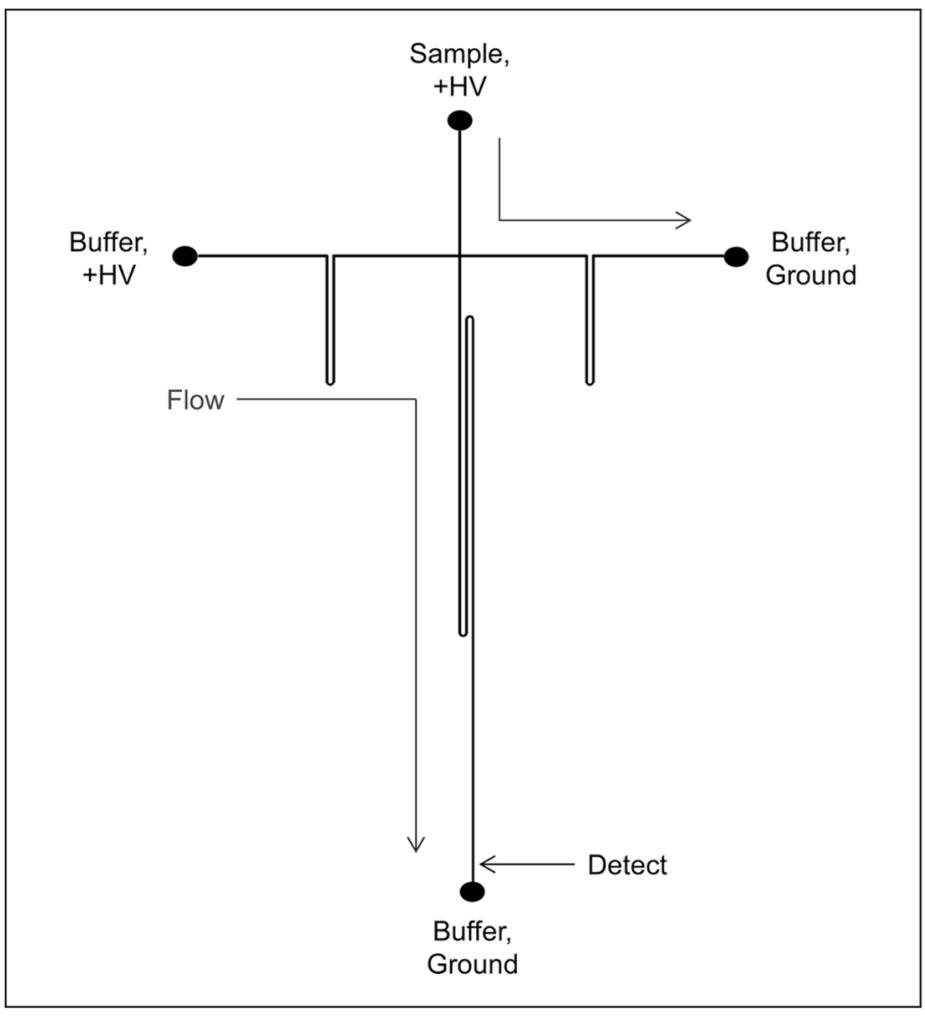

Figure 2.

Schematic of the microchip electrophoresis device. Channel dimensions were $15 \times 45 \mu \mathrm{m}$, and the separation distance was $14 \mathrm{~cm}$ (13 cm to detector). The sample reservoir side arm was $1 \mathrm{~cm}$ in length, whereas the buffer and sample waste side arms were $4 \mathrm{~cm}$ long. 


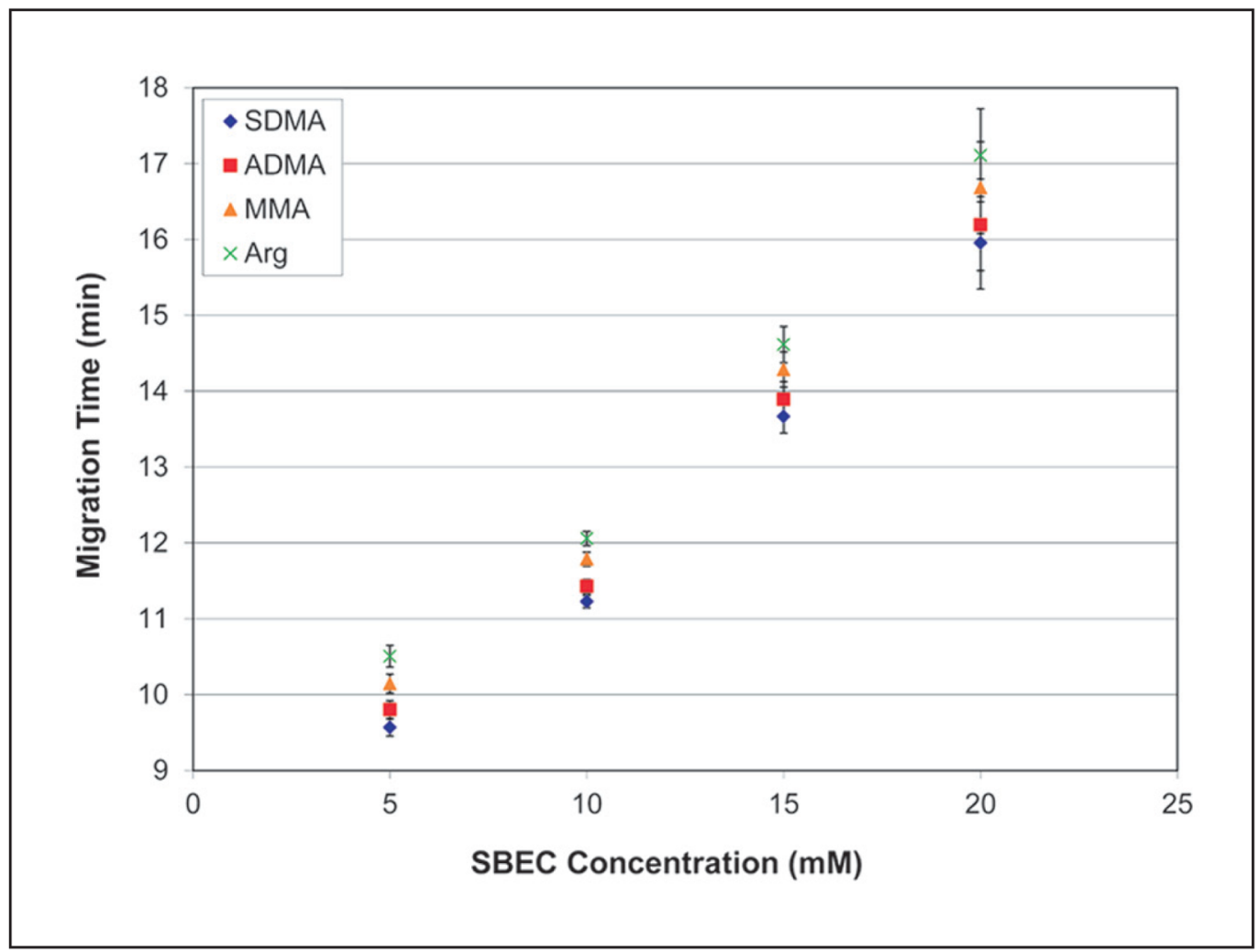

Figure 3.

The effect of sulfobutylether- $\beta$-cyclodextrin concentration on analyte migration times. The run buffer composition was $30 \mathrm{mM}$ pH 9.25 borate, and the separation voltage was $23 \mathrm{kV}$. 


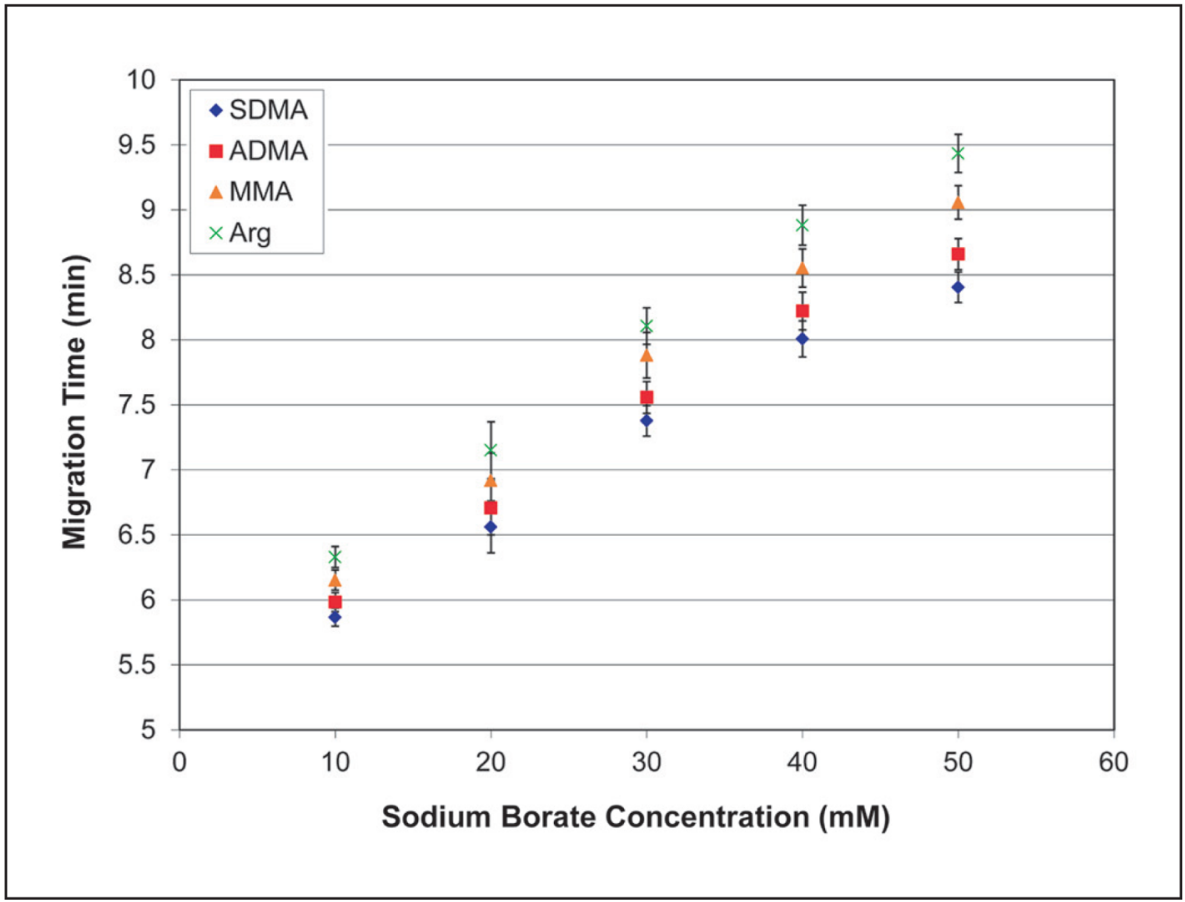

Figure 4.

The effect of sodium borate concentration ( $\mathrm{pH} 9.25)$ on analyte migration times. The run buffer composition was $5 \mathrm{mM}$ sulfobutylether- $\beta$-cyclodextrin, and the separation voltage was $28 \mathrm{kV}$. 


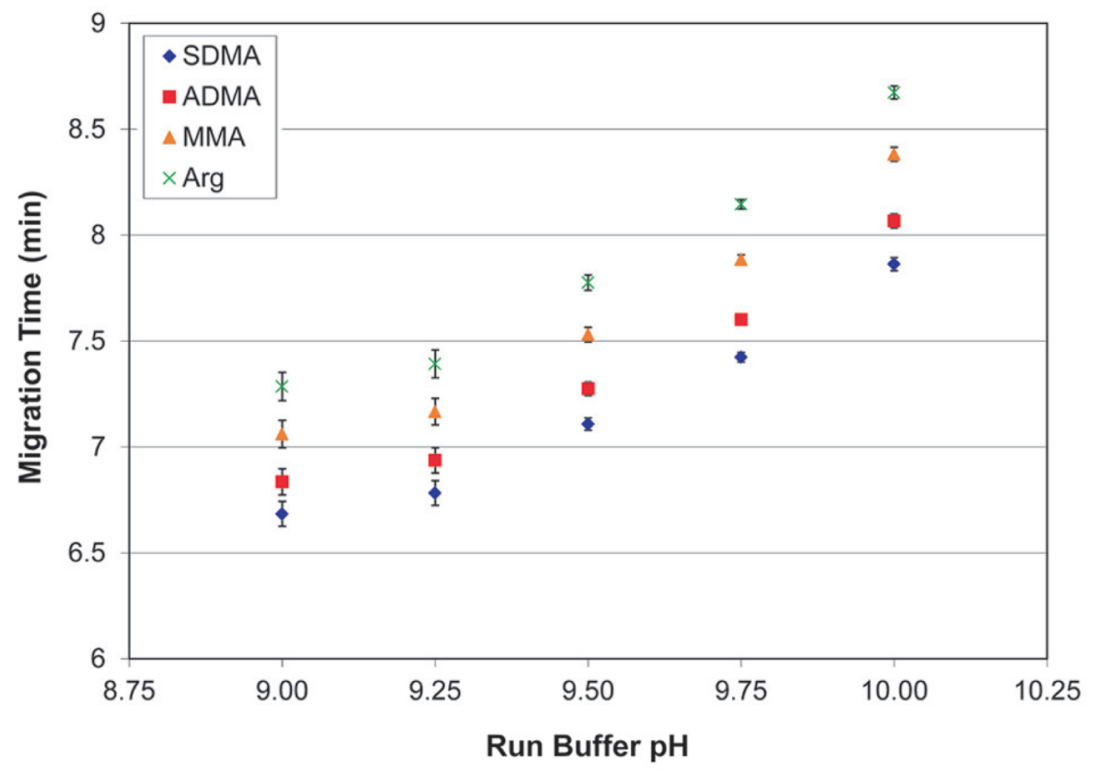

Figure 5.

The effect of run buffer $\mathrm{pH}$ on analyte migration times. The run buffer composition was 30 $\mathrm{mM}$ borate and $5 \mathrm{mM}$ sulfobutylether- $\beta$-cyclodextrin, and the separation voltage was $28 \mathrm{kV}$. 


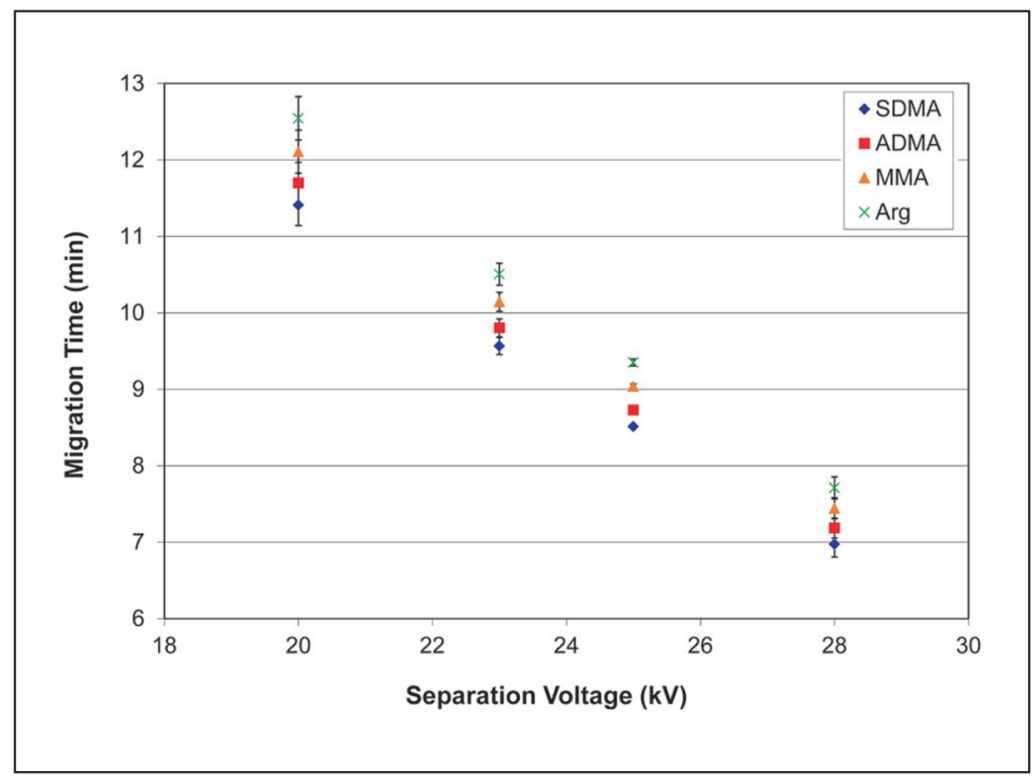

Figure 6.

The effect of applied separation voltage on analyte migration times. The run buffer contained $30 \mathrm{mM} \mathrm{pH} 9.25$ borate and $5 \mathrm{mM}$ sulfobutylether- $\beta$-cyclodextrin. 


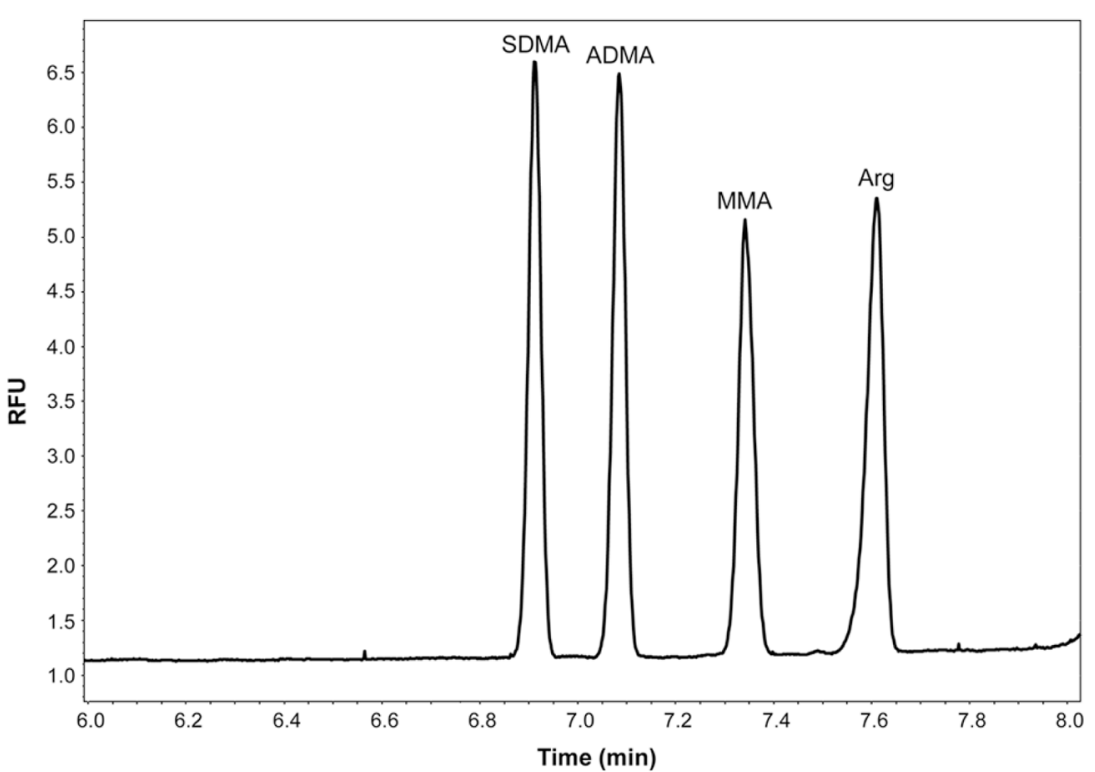

Figure 7.

Electropherogram depicting the optimized separation of the MAs with conventional capillary electrophoresis. A field strength of $430 \mathrm{~V} / \mathrm{cm}$ was applied across a $65 \mathrm{~cm}$ capillary $(50 \mathrm{~cm}$ to window). The run buffer contained $30 \mathrm{mM} \mathrm{pH} 9.25$ sodium tetraborate and $5 \mathrm{mM}$ sulfobutylether- $\beta$-cyclodextrin. 


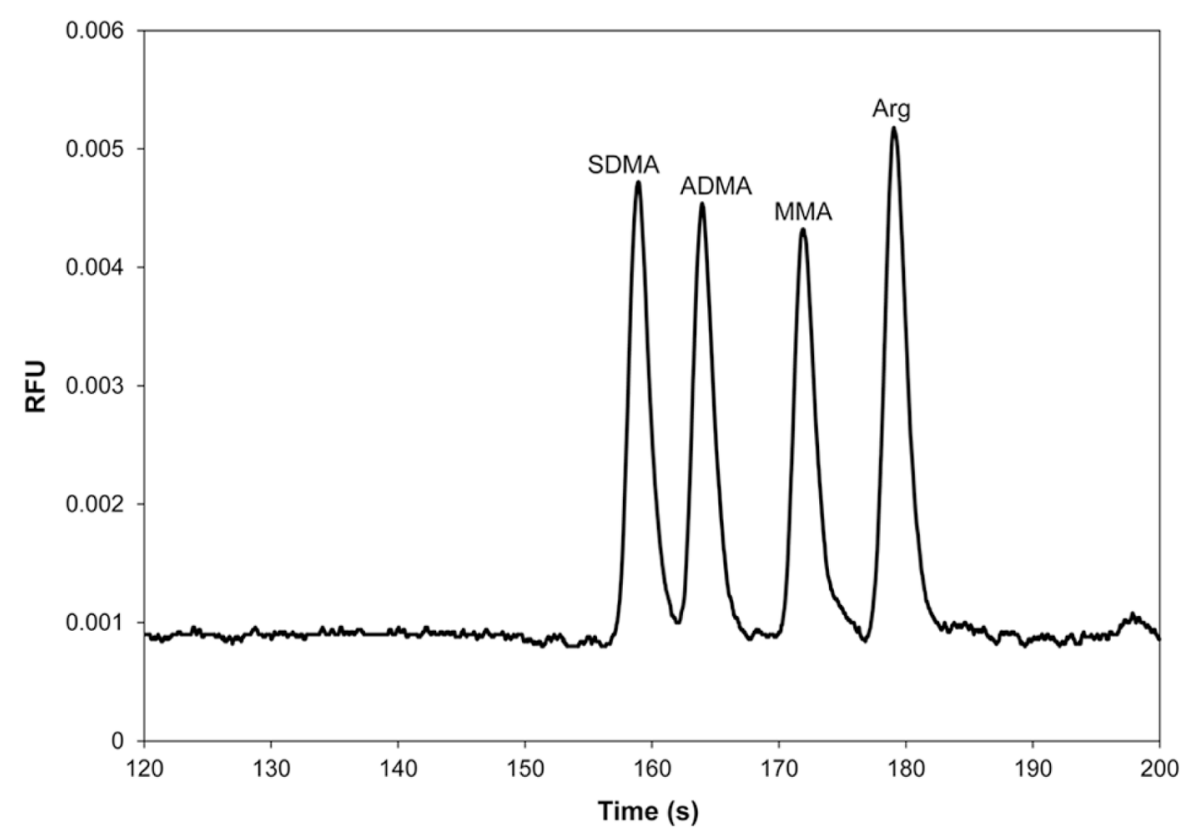

Figure 8.

Electropherogram depicting the separation of methylated arginines on a microchip electrophoresis device. Channel dimensions were $15 \times 45 \mu \mathrm{m}$ with a separation distance of $14 \mathrm{~cm}$ (13 cm to detector). The run buffer contained $30 \mathrm{mM} \mathrm{pH} 9.25$ sodium tetraborate and $5 \mathrm{mM}$ sulfobutylether- $\beta$-cyclodextrin. 


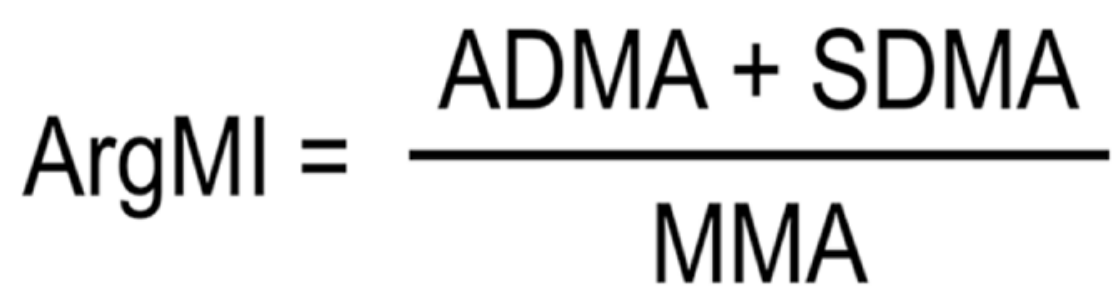

Figure 9. Equation 1

Arginine methylation index. 